\title{
Controle robusto aplicado na máquina de indução implementado no Matlab/Simulink
}

\author{
Robust control applied induction machine \\ employed of Matlab/Simulink
}

\author{
Ruberlei Gaino ${ }^{1}$; José Paulo Fernandes Garcia ${ }^{2}$; \\ Marcelo Carvalho Minhoto Teixeira ${ }^{3}$
}

\begin{abstract}
Resumo
Este trabalho refere-se à abordagem de uma estratégia/metodologia de controle para controle da máquina de indução em alta performance. O método clássico apresentado na literatura [1] compõe-se de controladores PID's atuando na estratégia de controle da velocidade da máquina. O modelo matemático da máquina de indução após um tratamento adequado, similarmente, conduz ao modelo matemático da máquina de corrente contínua, na qual a estratégia de controle é muito mais simples.

A proposta deste trabalho é a utilização de uma nova metodologia de controle que seja mais confiável e principalmente robusta e que atenda os requisitos de desempenho e estabilidade.

Será descrito a seguir que o método de Controle Robusto mostrou as condições de robustez, estabilidade e desempenho.

Palavras-Chave: Controle Robusto, LQG/LTR, Máquina de Indução, Coordenada de Campo.
\end{abstract}

\begin{abstract}
This work refers the approach of a control strategy/metodology for control of the induction machine in high performace. The classic method presented in the literature [1], they are composed of controllers PID's acting in the strategy of control of the speed of the machine. The mathematical model of the induction machine after an appropriate treatment, equaly leads to the mathematical model of the machine of continuous current, which the control strategy is much simpler. The proposal of this work was to use of a new control methodology that is more reliable and mainly robust and that assists the performance requirements and stability. It is be described that the method of Robust Control showed the robustness conditions, stability and performance.
\end{abstract}

Key words: Robust control, LQG/LTR, induction machine, field coordinate.

\footnotetext{
${ }^{1}$ Professor Assistente do Curso de Engenharia Elétrica, do C.T.U. da UEL

${ }^{2}$ Docente do Curso de Engenharia Elétrica da FEIS/UNESP

${ }^{3}$ Docente do Curso de Engenharia Elétrica da FEIS/UNESP
} 


\section{Introdução}

Inicialmente faremos o tratamento matemático da máquina de indução em Coordenadas de Campo com Corrente Imposta ao Estator [1], obtendo seu modelo para a referida aplicação da técnica de Controle Robusto.

- A discussão se aplica a motores de dois pólos, sendo que para máquinas de multipolo a velocidade é reduzida correspondentemente.

- Permeabilidade do ferro no estator e no rotor laminado é assumida infinita; saturação e perdas no ferro são desprezíveis.

O campo magnético no entreferro da máquina tem direção radial por causa das superfícies paralelas do rotor e estator e, considerando a permeabilidade infinita do ferro, os efeitos finais são desprezados, tratando-se do problema de campo magnético em apenas duas dimensões.

Considere-se a Figura 1, que representa o corte transversal da máquina de indução. Para visualizar e identificar as variáveis envolvidas no equacionamento da máquina, definem-se a seguir essas variáveis como sendo :

- $\alpha$ é a coordenada angular no estator, com referência na bobina 1 do estator. A posição de cada uma das bobinas é indicada em relação ao eixo magnético do enrolamento 1.

- Os centros dos enrolamentos 2 e 3 são idênticos e posicionados em $\alpha=\gamma=120$ graus e $\alpha=2 \gamma=240$, respectivamente.

- $\beta$ é o ângulo do rotor, agora com referência no eixo do enrolamento 1 do rotor.

- $\varepsilon(t)$ é o ângulo de rotação do motor, medido nas coordenadas do estator. - $\omega(\mathrm{t})=\left(\frac{\mathrm{d} \varepsilon}{\mathrm{dt}}\right)$ é a velocidade angular instantânea
do motor.

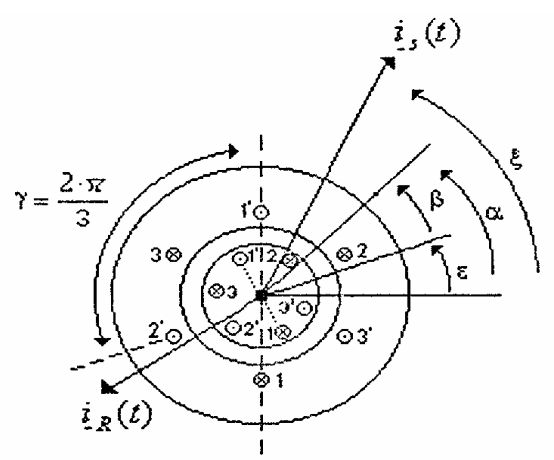

Figura 1 - Corte Transversal da Máquina de Indução.

$R_{s} \underline{\boldsymbol{i}}_{s}+L_{s} \frac{d \underline{\boldsymbol{i}}_{s}}{d t}+M \frac{d \underline{\underline{i}}}{d t} e^{j \varepsilon}+j \omega M \underline{\boldsymbol{i}}_{r} e^{j \varepsilon}=\underline{u}_{s}(t)$

$R_{r} \underline{i}_{r}+L_{r} \frac{d \underline{\boldsymbol{i}}_{r}}{d t}+M \frac{d \underline{\boldsymbol{i}}_{-s}}{d t} e^{-j \varepsilon}-j \omega M \underline{i}_{-s} e^{-j \varepsilon}=0$

$\left.J \frac{d \omega}{d t}=m_{d}(t)-m_{L}(t)=\frac{2}{3} \mathrm{~L}_{0} \operatorname{Im}\left[\underline{-}_{-s} \underset{-r}{(\underset{r}{*}} e^{j \varepsilon}\right) *\right]-m_{L}(\varepsilon, \omega, t) ;$

$\frac{d \varepsilon}{d t}=\omega$

sendo $R_{s}, R_{r}$ as resistências dos enrolamentos do estator e rotor, respectivamente;

$\mathrm{L}_{\mathrm{s}}, \mathrm{L}_{\mathrm{r}}$ e $\mathrm{M}$ a indutância própria do estator, do rotor e mútua;

$\underline{i}_{s}$ e $\underline{i}_{-r}$ os vetores correntes de estator e rotor;

$m_{d}$ e $m_{L}$ são os conjugados eletromagnético e de carga.

\section{Modelo do Motor de Indução em coordenadas de Campo}

Considerando como referência o vetor corrente de magnetização $i_{m R}$, a partir da Figura 2, podemos definir os vetores corrente $i_{s d}$ e $i_{s q}$ como a seguir:

$$
\begin{aligned}
& \left.i_{s d}=\operatorname{Re} \underline{\underline{i}}_{s} e^{-j p}\right\rfloor=i_{s} \cos \delta \\
& i_{s q}=\operatorname{Im}\left\lfloor\underline{i}_{s} e^{-j \rho}\right\rfloor=i_{s} \operatorname{sen} \delta
\end{aligned}
$$




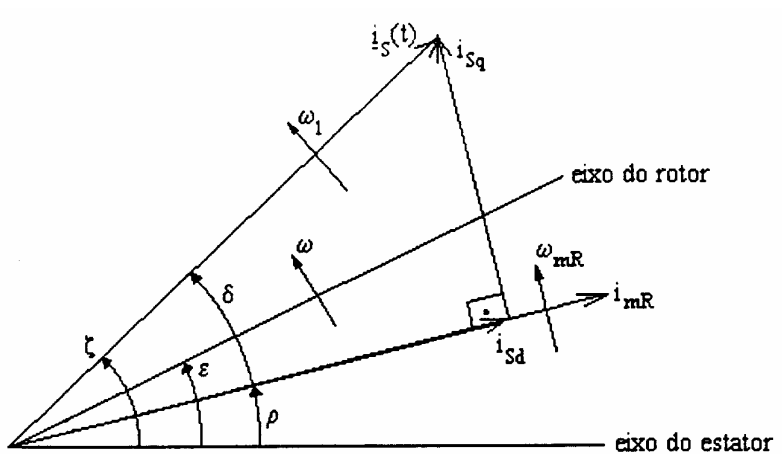

Figura 2 - Relações Angulares dos Vetores Correntes

Tendo como referência essas coordenadas ("coordenadas de campo"), podemos obter um conjunto de equações, dadas a seguir, [1].

$m_{D}(t)=k \quad i_{m_{R}} i_{s q}, k=\frac{2}{3} \frac{L_{0}}{1+\sigma}$

$T_{r} \frac{d\left(i_{m R}\right)}{d t}+j \omega_{m R} T_{r} i_{m R}+\left(1-j \omega T_{r}\right) i_{m R}=i_{-s} e^{-j p}$

$\frac{1}{\omega_{o}} \frac{d \rho}{d t}=\frac{\omega}{\omega_{o}}+\frac{i_{s q}}{\omega_{o} T_{r} i_{m R}}=\frac{\omega}{\omega_{o}}+\frac{\omega_{2}}{\omega_{o}}$

onde

$$
\frac{d \varepsilon}{d t}=\omega
$$

$\frac{d \rho}{d t}=\omega_{m R}(t)$

$\frac{d \xi}{d t}=\omega_{1}(t)=\omega_{m R}+\frac{d \delta}{d t}$

As correntes $i_{s d}$ e $i_{s q}$ podem ser obtidas pela transformação das correntes do estator a partir do ângulo de fluxo $\rho$. Realizando primeiramente a conversão das três correntes do estator para um sistema bifásico ortogonal, tem-se:

$\underline{i}_{-s}(t)=i_{s 1}+i_{s 2} e^{j \gamma}+i_{s 3} e^{j 2 \gamma}=i_{s a}+j i_{s b}$
Com a condição de correntes trifásicas balanceadas, $i_{s 1}+i_{s 2}+i_{s 3}=0$, tem-se:

$$
\begin{aligned}
& i_{s a}(t)=\frac{3}{2} i_{s 1}(t) \\
& i_{s b}(t)=\frac{\sqrt{3}}{2}\left[i_{s 2}(t)-i_{s 3}(t)\right]
\end{aligned}
$$

Essas correntes ca são novamente transformadas em coordenadas de campo, e convertidas em grandezas cc.

$\underline{i}_{s}(t) e^{-j p}=\left(i_{s a}+i_{i s b}\right)(\cos \rho-j \operatorname{sen} \rho)=i_{s d}+j_{i s q}$

Assim, tem-se:

$i_{\text {sd }}(t)=i_{\text {sa }} \cos \rho+i_{\text {sb }} \operatorname{sen} \rho$

$\mathrm{i}_{\mathrm{sq}}(\mathrm{t})=\mathrm{i}_{\mathrm{sb}} \cos \rho-\mathrm{i}_{\mathrm{sa}} \operatorname{sen} \rho$

A máquina assim equacionada apresenta uma estrutura semelhante à máquina $\mathrm{cc}$. Desse modo, uma estratégia de controle do torque poderá ser facilmente implementada, seguindo as estratégias utilizadas no controle das máquinas cc, por exemplo, a corrente magnetizante $i_{m R}$ pode ser mantida constante, em seu nível máximo, em operações em que a máquina esteja entre 0 e 1 p.u., sendo o torque controlado através da corrente em quadratura $i_{s q}$. Acima dessa faixa, pode-se diminuir $i_{m R}$, enfraquecendo o campo, obviamente, tendo-se o cuidado para não atingir valores elevados para $i_{s q}$.

\section{Controle Clássico do Motor de Indução em Coordenadas de Campo}

A função básica do esquema de controle é gerar correntes de referência para os conversores ca/ca, os 
quais efetivamente alimentarão o motor de indução. A Figura 3 apresenta um algoritmo de controle de velocidade apresentado em [1], dito aqui convencional, pois tem como principal atuador controlador PID. Pode-se observar que nesta Figura (utilizada para realização de simulações) não está representado o conversor, uma vez que o considerados como ideal em todas as simulações.

A estrutura de controle de velocidade da máquina de indução nesse caso é semelhante ao controle simplificado da máquina de corrente contínua: fixase a corrente de magnetização num valor constante através do bloco que contém o esquema de enfraquecimento de campo, em que $i_{m R}$ é mantida constante quando o motor está entre os valores nominais de velocidade (-1 a 1) pu. Fora dessa faixa de velocidade, $i_{m R}$ é variável. Assim, cria-se uma corrente de magnetização de referência. A partir da medida da velocidade do rotor, três controladores PID mantêm os valores de $i_{m R}, m_{d}$ e $\omega$ em seus valores de referência.

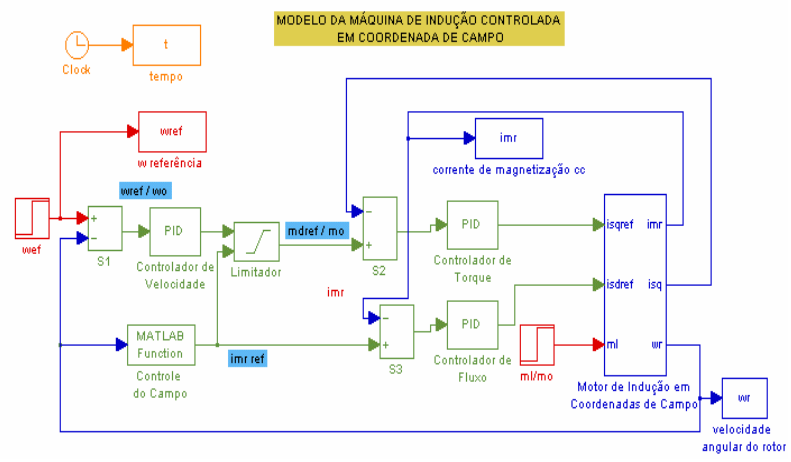

Figura 3 - Controle de Velocidade em Coordenadas de Campo.

O objetivo de enfraquecimento de campo é aumentar a velocidade a cargas reduzidas, admitindo a desvantagem de aumento da corrente de armadura a um determinado torque, portanto não é apropriado empregar o enfraquecimento de campo, a não ser que as possibilidades do controle de armadura tenham sido completamente exploradas.

Claramente, o princípio de controle em coordenada de campo parece um caminho efetivo de desacoplamento da estrutura de controle multivariável complexo da máquina de indução.

No entanto, um detalhe importante é que, para obter os sinais de referência, os controladores PID necessitam das estimativas de $i_{m R}$ e $i_{s q}$. Este fato pode tornar bastante complicado o esquema de controle dito aqui convencional. Esta complicação não aparece no esquema de controle LQG/LTR que vamos propor.

\section{Método "Linear Quadratic Gaussian/Loop Transfer Recovery (LQG/LTR)": pontos principais}

O objetivo desse item é a apresentação resumida do método LQG/LTR. Todas as informações foram tomadas a partir das referências [2] e [3], onde estão mais detalhadas.

As características mais importantes deste método podem ser citadas a seguir.

- A robustez do controlador em face de uma ampla classe de erros de modelagem é garantida pelo procedimento;

- A técnica foi concebida para aplicação em sistemas multivariáveis;

- O procedimento do projeto é de natureza sistemática;

- A metodologia se baseia numa abordagem freqüencial, aplicando-se assim a sistemas lineares e invariantes no tempo;

- O número de parâmetros de projeto é relativamente pequeno;

- Existe software de apoio ao projeto disponível.

O propósito é apresentar uma metodologia de projeto que permita a determinação de um compensador, a ser localizado no ramo direto da malha de controle, de maneira que sejam satisfeitos os requisitos de desempenho e estabilidade.

Os instrumentos mais importantes utilizados para o desenvolvimento dessa metodologia são o 
Regulador Linear Quadrático (RLQ) e o filtro de Kalman (FK).

Com relação ao desempenho, serão considerados como objetivos a serem atendidos pelo sistema real:

- acompanhamento do sinal de referência;

- rejeição de perturbação externa;

- A insensibilidade à variação da planta;

- A rejeição do erro de medida;

O compensador deverá ser estável para qualquer sistema real.

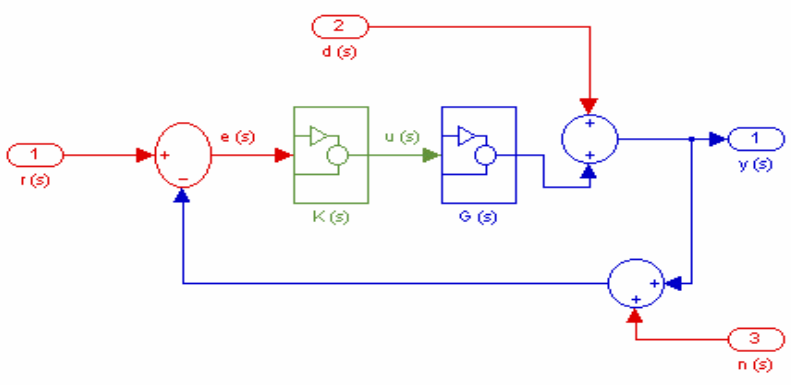

Figura 4 - Diagrama de Blocos

Um diagrama esquemático pode ser visualizado na Figura 5. Sendo $\mathrm{C}^{m}$ o conjunto de vetores complexos, Cmxm, o conjunto de matrizes complexas $\mathrm{m} \times \mathrm{m}$, a seguir segue as definições de cada variável representada nesta Figura:

- $\mathrm{r}(\mathrm{s}) \in \mathrm{C}^{m}$ é o sinal de referência;

- $\mathrm{e}(\mathrm{s}) \in \mathrm{C}^{m}$ é o sinal de erro;

- $\mathrm{u}(\mathrm{s}) \in \mathrm{C}^{m}$ é o sinal de controle (ou de entrada);

- $\mathrm{d}(\mathrm{s}) \in \mathrm{C}^{m}$ é a perturbação refletida na saída da planta;

- $\mathrm{y}(\mathrm{s}) \in \mathrm{C}^{m}$ é o sinal de saída;

- $\mathrm{n}(\mathrm{s}) \in \mathrm{C}^{m}$ é o erro de (ruído) de medida.

Definem-se também as matrizes de funções de transferência:

- $\mathrm{K}(\mathrm{s}) \in \mathrm{C}$ mxm é a matriz de funções de transferência do compensador;
- $\mathrm{G}_{\mathrm{n}}(\mathrm{s}) \in \mathrm{C}^{m x m}$ é a matriz de funções de transferência da planta;

- $\mathrm{G}_{\mathrm{n}}(\mathrm{s}) \mathrm{K}(\mathrm{s}) \in \mathrm{C}^{m x m}$ é a matriz de funções de transferência de malha aberta ou matriz de funções de transferência do ramo direto;

- $\mathrm{C}(\mathrm{s})=\left[\mathrm{I}+\mathrm{G}_{\mathrm{n}}(\mathrm{s}) \mathrm{K}(\mathrm{s})\right]^{-1} \mathrm{G}_{\mathrm{n}}(\mathrm{s}) \mathrm{K}(\mathrm{s}) \in \mathrm{C}^{m x m}$ é a matriz de funções de transferência de malha fechada.

A aplicação da metodologia determina o compensador $\mathrm{K}(\mathrm{s})$ tal que $\mathrm{G}_{\mathrm{n}}(\mathrm{jw}) \mathrm{K}(\mathrm{jw})$ encaixa-se entre as barreiras de desempenho e estabilidade conforme é mostrado na Figura 5.

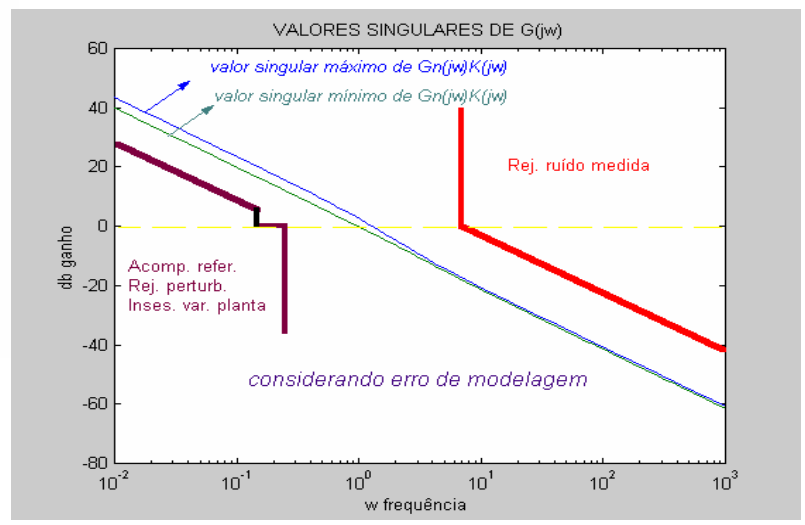

Figura 5 - Barreiras de robustez do desempenho e da estabilidade

\section{Aplicação da Metodologia LQG/LTR no Controle do Motor de Indução}

As equações da máquina em coordenadas de campo estão apresentadas a seguir

$$
i_{s d}=T_{r}\left(\frac{d i_{m R}}{d t}\right)+i_{m R}
$$

$$
i_{s q}=\left(\omega+\omega_{2}\right) T_{r} i_{m R}
$$

$$
m_{d}=\frac{2}{3} \frac{L_{o}}{1+\sigma_{r}} i_{m R} i_{s q}
$$


Se a magnitude da corrente de magnetização, bem como o fluxo, são mantidos constantes, então este conjunto de equações pode ser linearizado considerando os passos a seguir. Seja $\mathrm{v}_{\mathrm{sq}}=\mathrm{K}_{\mathrm{i}}\left(\mathrm{i}_{\mathrm{sq}}^{*}-\mathrm{i}_{\mathrm{sq}}\right)$, onde $\mathrm{K}_{\mathrm{i}}$ é o ganho de corrente e a componente da tensão $\mathrm{v}_{\mathrm{sq}}$ é a tensão entregue ao estator transformado no controlador de corrente para chaveamento do inversor:

$v_{q s}=R_{s} i_{q s}+\sigma L_{s} \frac{d i_{q s}}{d t}+L s \omega_{1} i_{d s}$

onde $\sigma$ fluxo de dispersão do motor.

Portanto o controle desacoplado de corrente será

$\sigma L_{s} \frac{d i_{s q}}{d t}+R_{s} i_{s q}=v_{s q}-\frac{L_{s}}{L_{r}} R_{r} i_{s q}^{*}-L_{s} i_{s d} \omega$

A dinâmica do motor pode ser expressa como

$J_{m} \frac{d \omega_{m}}{d t}+B_{m} \omega_{m}=m_{d}-m_{L}$

Considerando $i_{s d}$ constante [4], o modelo equivalente pode ser transformado numa função de transferência de segunda ordem.

$G_{p}(s)=\frac{\omega_{m}(s)}{i_{s q}^{*}(s)}$

$G_{p}(s)=\frac{K^{\prime}}{\left(s \tau_{s}+1\right)\left(s \tau_{m}+1\right)}$

sendo $K^{\prime}=\frac{(K i-K f) K t}{K e}$ a constante equivalente do motor e $\tau_{e}, \tau_{m}$ são as constantes elétrica e mecânica respectivamente.

O controle desacoplado de corrente junto com a equação dinâmica da máquina estabelece o modelo equivalente apresentado na Figura 6.

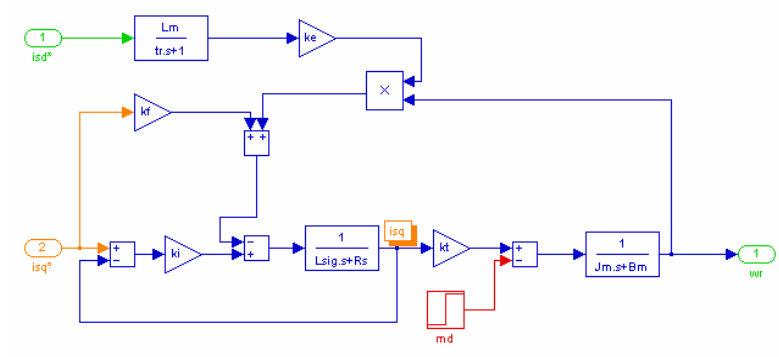

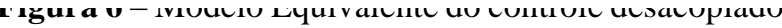
de corrente do motor de indução.

Procede-se agora aos dados numéricos para o modelo da planta linearizada e obtêm as matrizes linearizadas do sistema.

$$
A=\left[\begin{array}{cc}
-4.5 & -2.38 \\
1 & 0
\end{array}\right] ; B=\left[\begin{array}{ll}
1 & 0
\end{array}\right]^{T} ; C=[0
$$

\section{Aplicação da Metodologia LQG/LTR}

Resulta-se com isso o modelo da planta nominal citado no item anterior, portanto, de acordo com o LQG/LTR, projeta-se o compensador.

Desenvolvendo os resultados a seguir no programa para obter as matrizes do compensador, ou seja, a obtenção da matriz de ganho do observador $\boldsymbol{H}$ e a matriz ganho de realimentação $\boldsymbol{G}$.

A seguir visualiza-se através do gráfico o comportamento do controlador projetado para análise do seu desempenho.

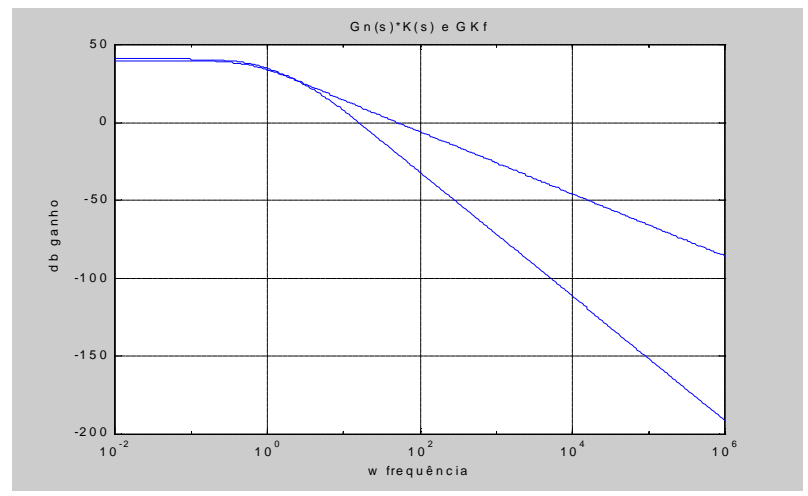

Figura 7 - Aplicação da Metodologia LGQ/LTR. 
Quando $\rho \rightarrow 0^{+}$o sistema em malha fechada $G_{N}(s) K_{s}(s)$, se aproxima do sistema $G K f$, das baixas para as altas freqüências.

\section{Resultados Obtidos em simulações e comparações com o controle via PI}

Neste segmento serão apresentados os resultados na forma gráfica obtidos a partir de diversas simulações e situações. Deve-se ressaltar os seguintes pontos durante as análises:

(i) as simulações do motor controlado com estratégia PI consideram acessíveis as variáveis $i_{m R}, i_{s q} e \omega$. Obviamente, na prática, a estimativa de $i_{m R} e i_{s q}$ não é uma tarefa fácil e nem sempre exequíivel;

(ii) as simulações do motor controlado com estratégia LQG/LTR considera acessível somente a variável $\omega$ (velocidade angular do rotor). Essa variável é perfeitamente mensurável.

Diante do que foi dito em (i) pode-se considerar que os resultados obtidos nas simulações para o caso em que o motor é controlado por PI são apenas teóricos e otimistas, uma vez que escondem uma grande dificuldade: a estimativa de $i_{m R} e i_{s q}$. Já os resultados obtidos no caso em que o motor é controlado através de estratégia LQG/LTR são resultados que podem ser perfeitamente obtidos na prática.

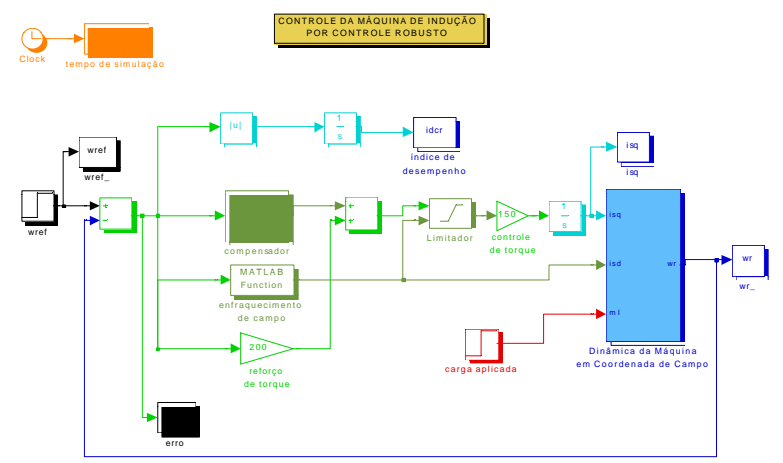

Figura 8 - Esquema de Controle de Velocidade com LQG/LTR.

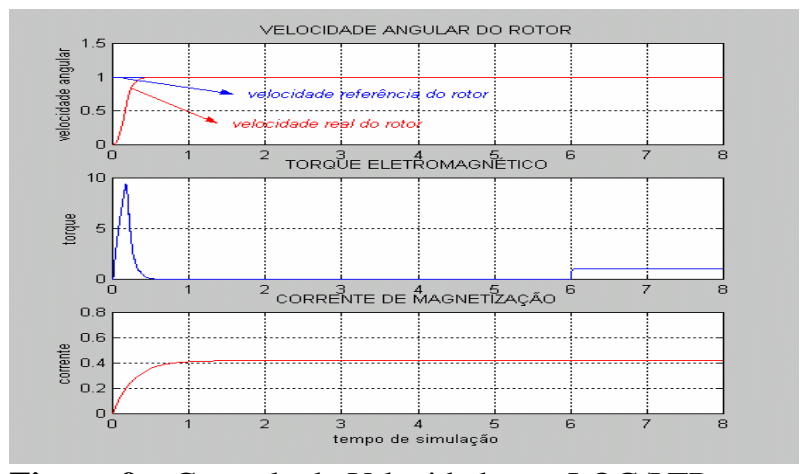

Figura 9 - Controle de Velocidade por LQG/LTR.

Na estrutura mostrada na Figura 8, pode-se notar o compensador projetado para o controle da corrente $i_{s q}$, o bloco que mantém a corrente $i_{s d}$ constante, o limitador de sinal e o bloco de reforço de torque. Com essa estrutura e aquela referente ao controle dito convencional Figura 3, foram realizadas seqüências de vinte simulações, com seis parâmetros da máquina variando simultaneamente e aleatoriamente em cada simulação. Em cada seqüência de vinte simulações contempla-se a seguinte situação:

i) Partida a vazio com posterior aplicação de carga de mesmo valor para as vinte simulações;

ii) Partida a vazio com posterior aplicação de carga aleatória para cada uma dbbas vinte simulações;

iii) Partida a vazio com reversão de velocidade em 5 segundos e aplicação de carga após a reversão.

Os resultados são mostrados em forma gráfica, snde plota-se o índice de desempenho dado por:

$I D=\int|e(t)| d t$ sendo $e(t)=\omega(t)_{\text {ref }}-\omega(t)_{\text {motor }}$

O objetivo desse índice é tornar exata a comparação entre os desempenhos das duas estratégias estudadas: convencional (com PI) e LQG/ LTR. Obviamente quanto menor for o valor numérico do índice, melhor terá sido o comportamento dinâmico das respostas. 
- Partida a vazio com posterior aplicação de carga de mesmo valor para as vinte simulações

O gráfico, a seguir, da Figura 10 mostra a norma das variações dos parâmetros da máquina em torno dos valores nominais. Assim, na primeira simulação de cada série, a norma é igual a zero, ou seja, simulouse a máquina com seus valores nominais. A partir da segunda simulação até a vigésima forçou-se os seis parâmetros da máquina a assumirem (de forma aleatória) valores fora do nominal. Obviamente os controladores foram mantidos constantes em todas as simulações, com seus valores obtidos a partir do projeto, considerando a máquina com seus valores nominais.

O segundo gráfico da Figura 10 mostra o resultado do índice de desempenho correspondente a cada simulação feita com controladores PI. Finalmente o terceiro gráfico da Figura 10 apresenta o valor numérico do índice de desempenho correspondente a cada uma das vinte simulações realizadas, utilizando-se a estratégia de controle LQG/LTR.

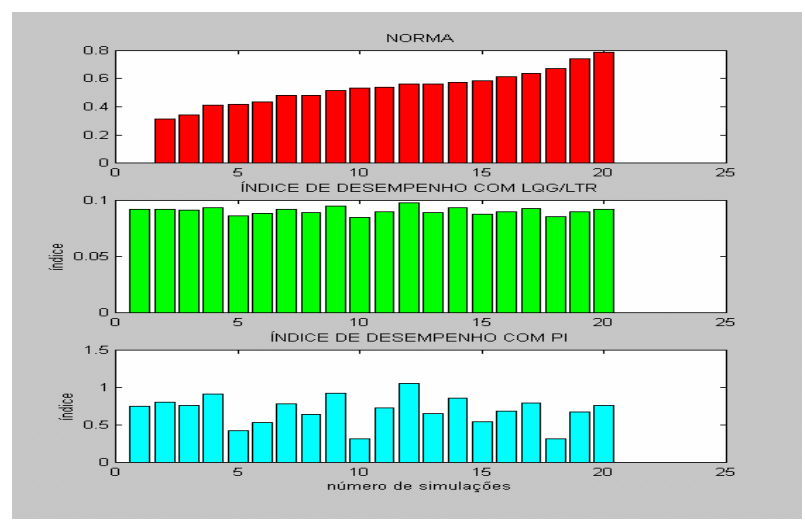

Figura 10 - Gráfico de Desempenho do Sistema com Carga Constante em vinte simulações realizadas.

Podemos notar, pela análise dessas figuras, que, para todas as simulações, os índices de desempenho foram bem menores para o controle com LQG/LTR (em torno de 0.1) do que os índices obtidos com controle PI (em torno 0.6), mostrando-se o sistema muito mais sensível às variações paramétricas neste último.
A Figura 11, a seguir, mostra as respostas no tempo de uma das vinte simulações realizadas. $\mathrm{Na}$ primeira curva plota-se a velocidade de referência juntamente com a velocidade real do motor, utilizando-se LQG/LTR. Em seguida, mostra-se também a evolução no tempo do sinal do erro e do índice de desempenho. A Figura 12 mostra as respostas no tempo do motor controlado com PI para a mesma simulação.

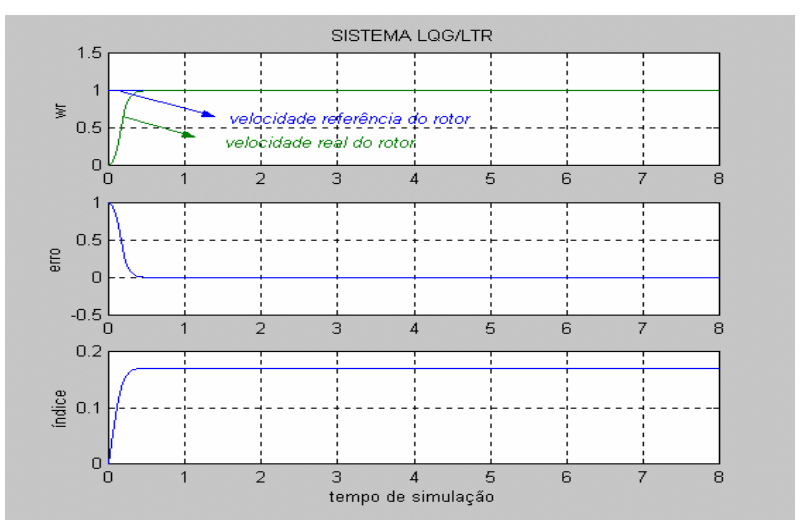

Figura 11 - Respostas no tempo: Sistema de Controle LQG/LTR com Carga Constante.

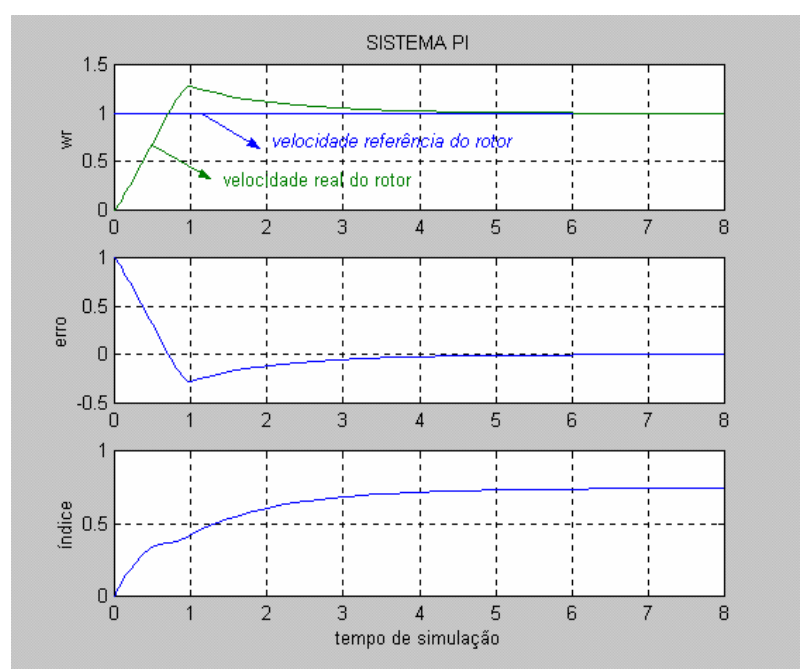

Figura 12 - Respostas no tempo: Sistema de Controle PI com Carga Constante. 


\section{- Partida a vazio com posterior aplicação de carga aleatória em cada uma das vinte simulações}

Passa-se ao caso em que a carga não é mais a mesma em todas as simulações: com o programa de variação paramétrica aleatória do sistema, acrescenta-se agora a variação da carga que se alterará de valor a cada iteração.

As respostas são apresentadas graficamente, na mesma forma apresentada no caso anterior: Figura 13 apresenta as normas das variações paramétricas em cada série de vinte simulações; seus respectivos índices de desempenho para os dois tipos de controladores utilizados.

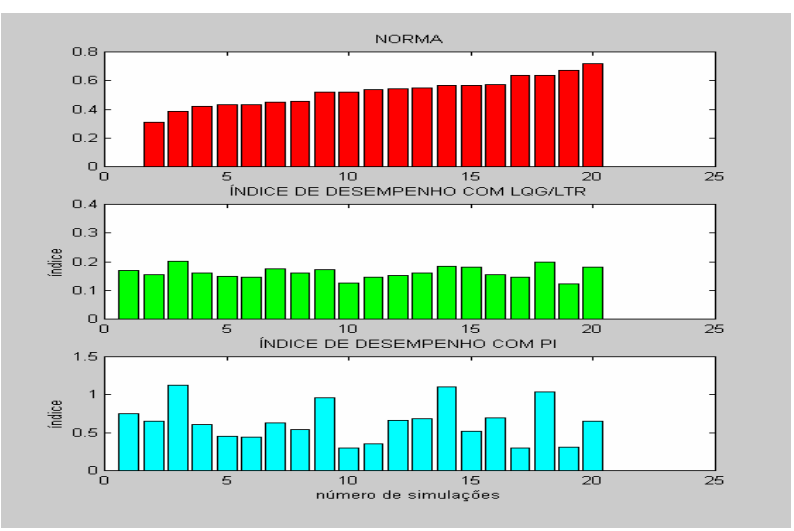

Figura 13 - Gráfico de Desempenho do Sistema com carga variável aplicada aleatoriamente em cada uma das vinte simulações.

Novamente os índices de desempenho indicam claramente melhor desempenho dinâmico e menor sensibilidade para o controle LQG/LTR. As respostas no tempo para uma das quarenta simulações são apresentadas a seguir, Figura 14 caso LQG/LTR e Figura 15 caso PI.

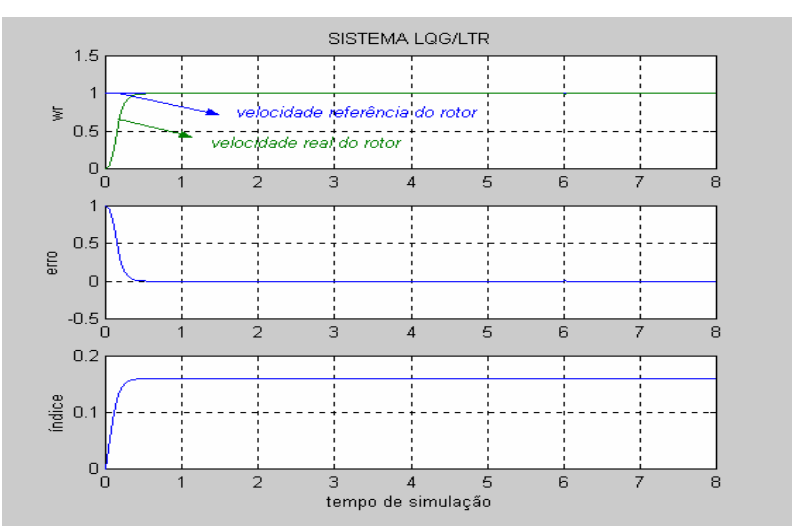

Figura 14 - Respostas no tempo: Sistema de Controle LQG/LTR com carga aplicada aleatoriamente.

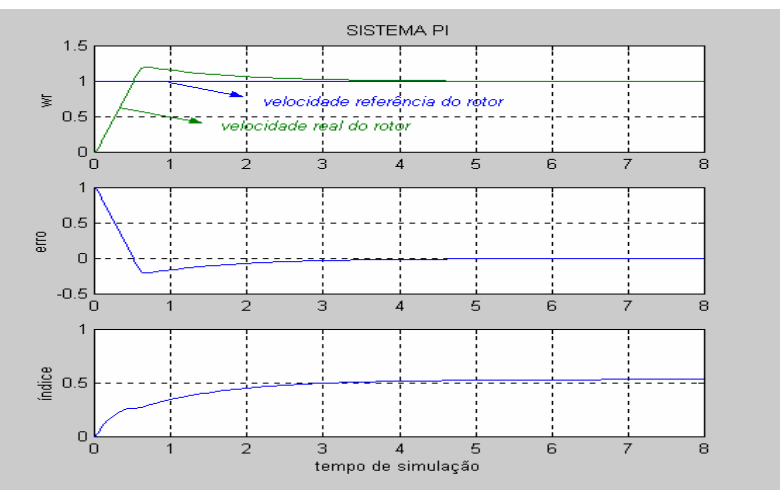

Figura 15 - Respostas no tempo: Sistema de Controle PI com carga aplicada aleatoriamente.

\section{- Partida a vazio com reversão de velocidade em} 5 segundos e aplicação de carga após a reversão

Nesta nova seqüência de simulações, teremos a reversão de velocidade em 5.0 segundos com aplicação de carga após a reversão. Serão apresentados os resultados seguindo as mesmas formas gráficas apresentadas anteriormente.

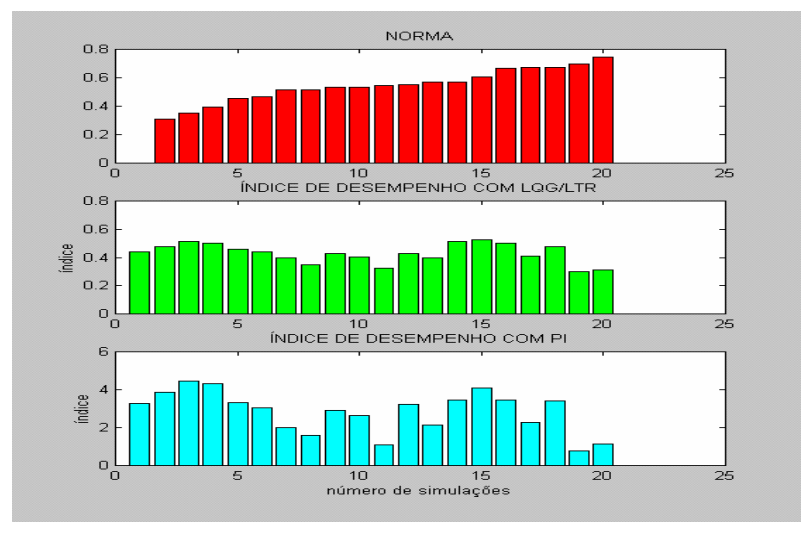

Figura 16 - Gráfico de Desempenho do Sistema com reversão de velocidade.

Observa-se que neste caso, com reversão de velocidade, o índice de desempenho do sistema PI, foi o pior dentre os três casos, não tendo uma resposta rápida transitória, enquanto que no sistema LQG/ LTR, obteveram-se respostas dos índices, semelhantes aos casos anteriores. A seguir as respostas no tempo de uma das simulações deixa claro o melhor desempenho dinâmico do motor quando controlado por LQG/LTR. 


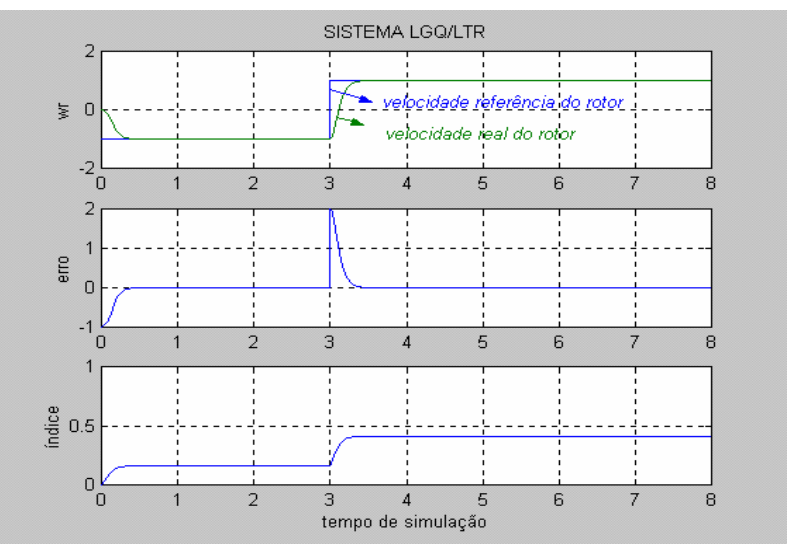

Figura 17 - Respostas no tempo: Sistema de controle LQG/LTR com reversão de velocidade.

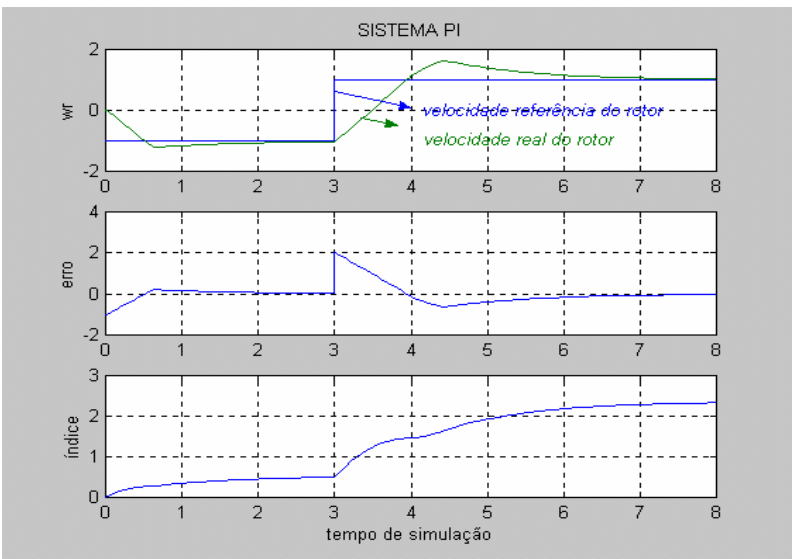

Figura 18 - Respostas no tempo: Sistema de controle PI com reversão de velocidade.

Os resultados obtidos nas diversas situações anteriores partiram de projetos cujos controladores foram projetados de tal forma que a corrente isq não ultrapassasse valores limites. Dentro da estratégia de projeto LQG/LTR os valores limites ficaram em torno de 15 p.u. durante os transitórios. Tais valores são, de fato, relativamente elevados. Abordamos a seguir uma estratégia para garantir que um determinado nível de pico não seja ultrapassado, através de alterações no algoritmo da estrutura de controle.

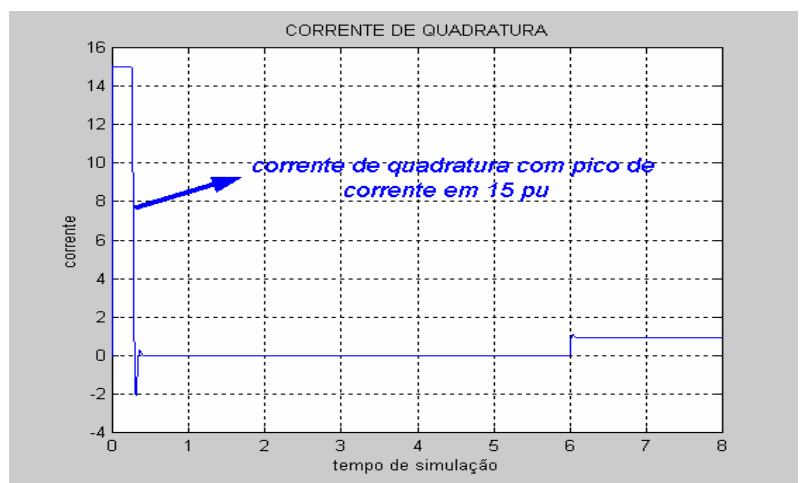

Figura 19 - Pico da corrente.

Admitindo que a corrente atinja um valor máximo de até cinco vezes o valor nominal, para realizar este ajuste, incluiu-se no ramo direto a estrutura mostrada na Figura 20, com o intuito de se diminuir o tempo em que a corrente fique na amplitude máxima. $\mathrm{O}$ resultado desta inclusão piorou o índice de desempenho, porém a estratégia seguida foi o aumento do valor da corrente de magnetização de 0.42 pu para $0.92 \mathrm{pu}$, colocando novamente o índice de desempenho em valores iguais aos anteriores. A seguir os resultados simulados foram plotados nas figuras a seguir.

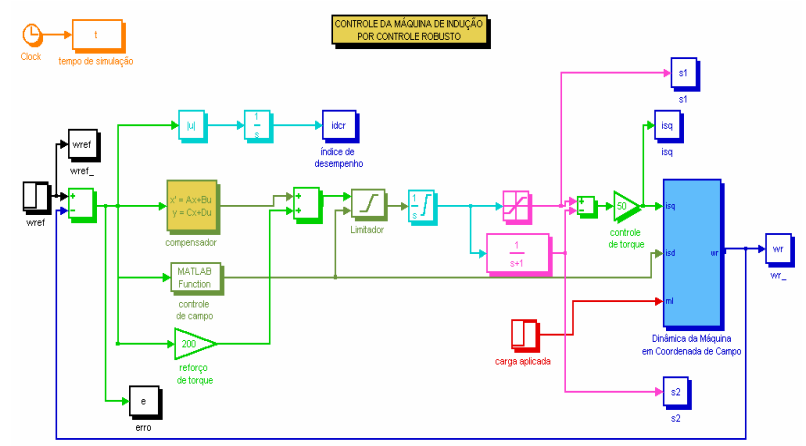

Figura 20 - Sistema LQG/LTR com inclusão de saturador e realimentação subtrativa 


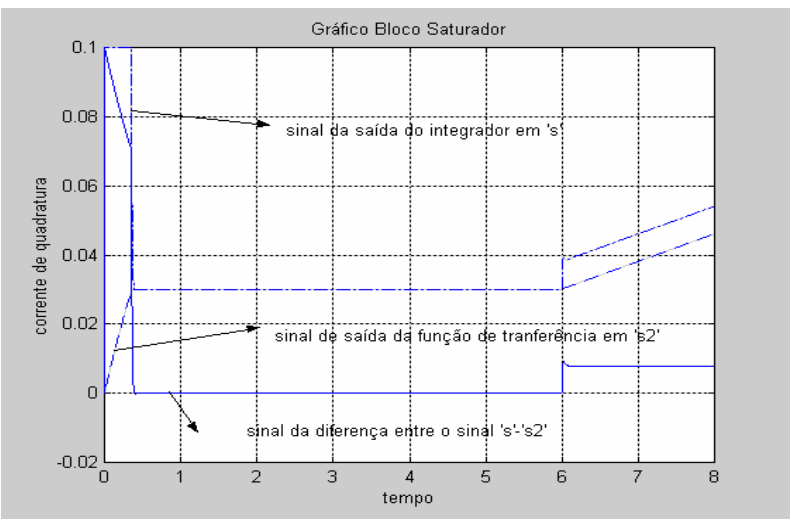

Figura 21 - Comportamento da Corrente de Quadratura com a inclusão do Bloco Saturador.

Devido à limitação na corrente de quadratura para níveis mais adequados, limitou-se o nível de integração, ocorrendo uma instabilidade, o recurso foi inserir em paralelo uma função de transferência que gera um sinal de mesmo comportamento que o sinal instável; a subtração desses sinais torna o sinal estável novamente, sem afetar o seu comportamento. Com este bloco saturador, piorou o índice de desempenho e o recurso necessário foi o aumento do valor da corrente de magnetização, $(0.92 \mathrm{pu})$.

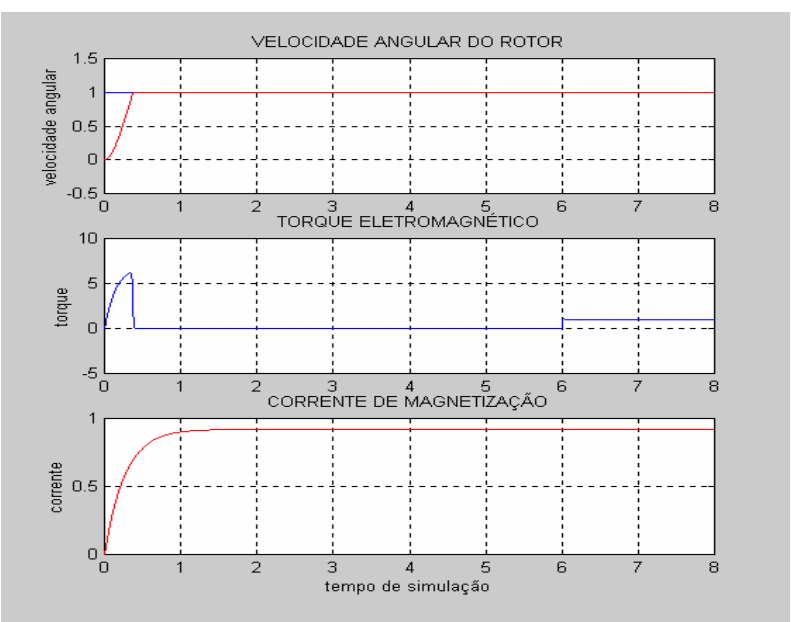

Figura 22 - Gráfico do sistema LQG/LTR com inclusão do saturador: velocidade de referência 1 p.u.

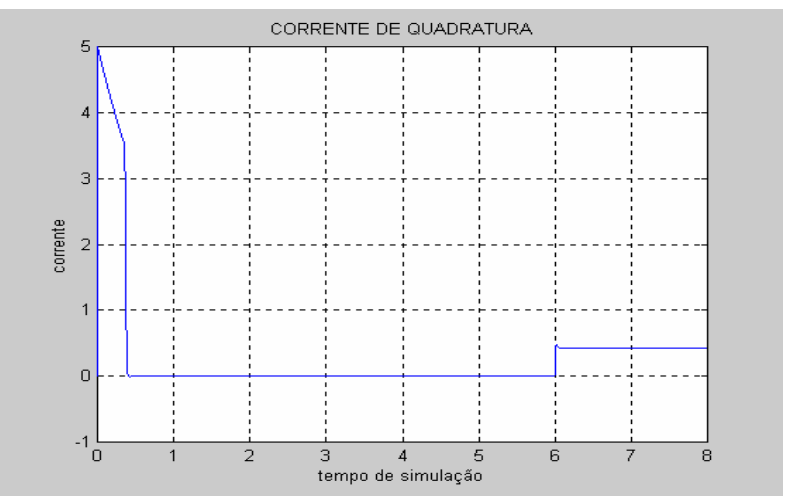

Figura 23 - Sistema LQG/LTR com saturador

Os resultados anteriores foram obtidos para velocidade de referência nominal (1 p.u.). Os resultados para o sistema a baixa velocidade ( 0.4 p.u.) também foram bons, como mostram as figuras a seguir.

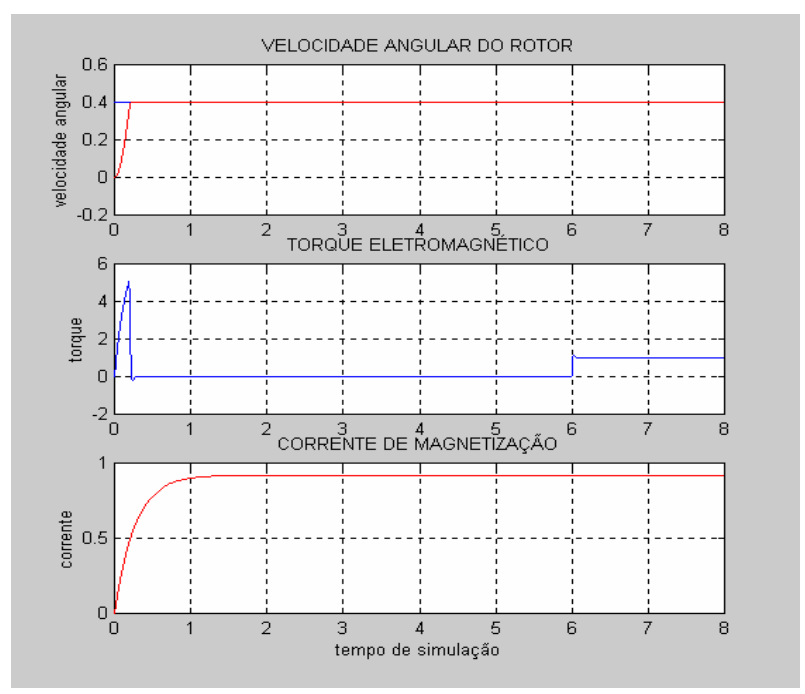

Figura 24 - Sistema LQG/LTR com saturador.

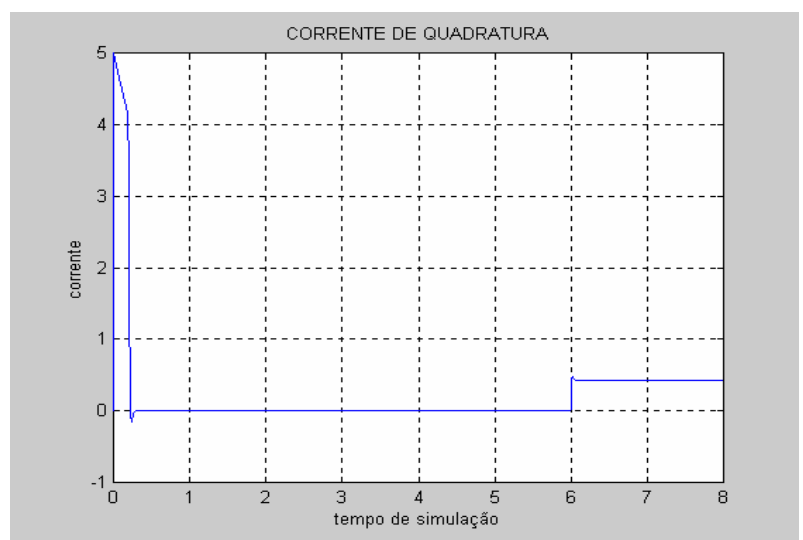

Figura 25 - Sistema LQG/LTR com saturador. 
Também foram incluídos testes para reversão de velocidade, cujos resultados são mostrados a seguir.

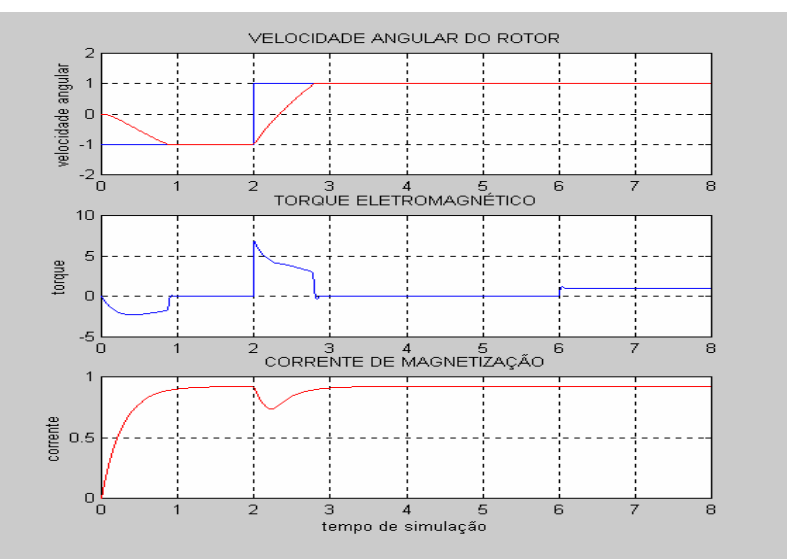

Figura 26 - Sistema LQG/LTR com saturador.

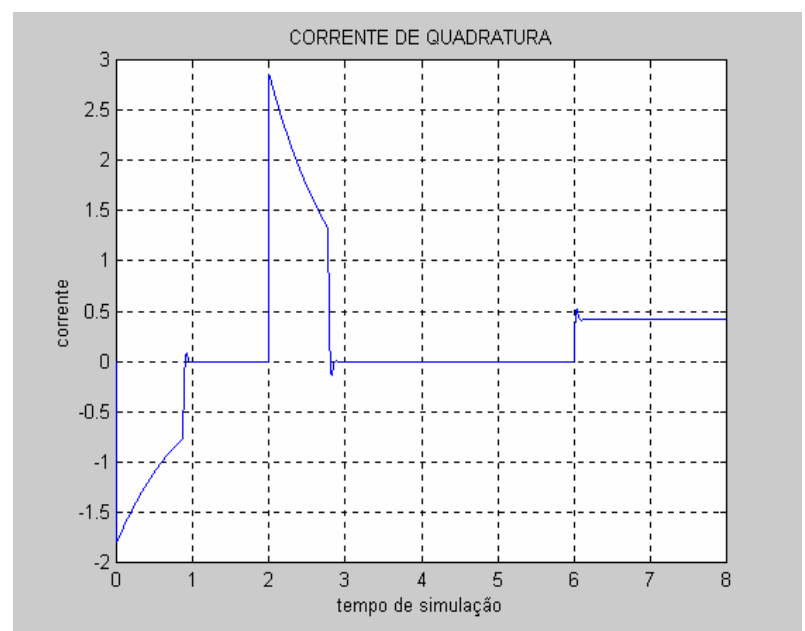

Figura 27 - Sistema LQG/LTR com saturador.

Para uma série de vinte simulações, com alteração simultânea e aleatória de seis parâmetros (considerando o bloco saturador, os índices de desempenhos são mostrados a seguir, sendo que esses gráficos referem-se a carga constante e rotação da máquina em um único sentido, Figura 28 , carga variável em um único sentido, Figura 29 , reversão de velocidade com aplicação de torque de carga constante, Figura 30.

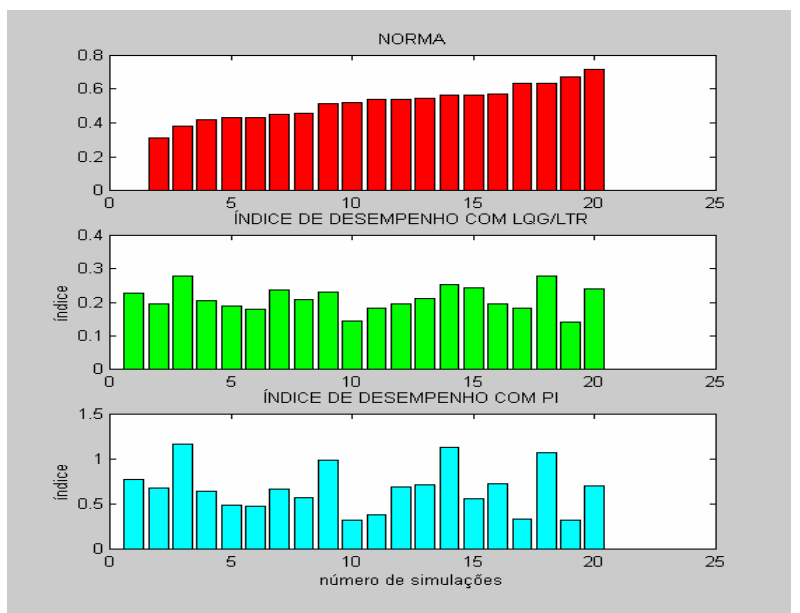

Figura 28 - Gráfico do Desempenho do sistema com saturador e carga constante.

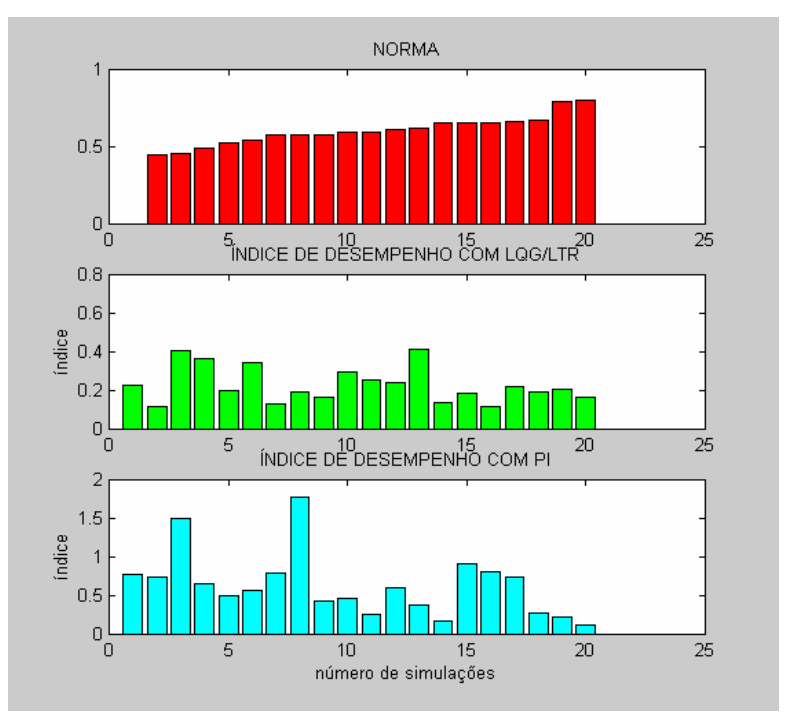

Figura 29 - Gráfico do Desempenho do sistema com saturador e carga variável.

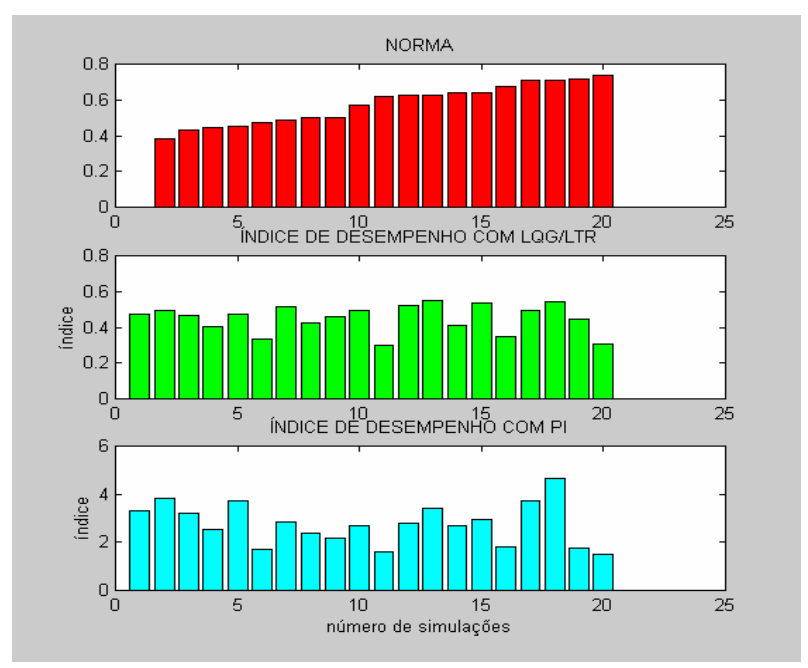

Figura 30 - Gráfico do Desempenho do sistema com saturador reversão de velocidade e aplicação de carga. 
Observa-se que o índice de desempenho das respostas para o motor controlado com LQG/LTR, com a restrição imposta, aumentou em comparação aos casos anteriores, sem restrição por saturador (de 0.1 para algo em torno de 0.2 ), no entanto, ainda supera o controle efetuado por PI.

\section{Conclusões}

Um resultado importante foi que se obteve uma metodologia de controle mais adequada do que apresentado em [1], no que se refere ao controle PID.

A técnica apresentada em [1] para cada modelo, foi o ajuste do PI, em que se observou a dificuldade do ajuste simultâneo dos três PI's e a precisão, comprometendo a velocidade da resposta do sistema.

Os problemas observados com os PI's, além do processo do ajuste ser empírico, foram as oscilações indesejadas que são introduzidas na dinâmica da máquina. Além disso, outro problema a ser citado é que existe a necessidade de estimar parâmetros $\left(i_{m R}, i_{s q}\right)$, para criar as referências no modelo de controle com PID.

A estrutura com a metodologia LQG/LTR é simplificada e de fácil ajuste, além de não haver necessidade de fazer qualquer estimativa dos parâmetros da máquina.

Os índices de desempenho comprovaram que o sistema com LQG/LTR superam os índices com PID, sendo assim ficou evidente um melhor desempenho da desta metodologia.

É importante salientar que consideramos o conversor, controlado por corrente, e por isso a nossa análise se restringiu à comparação qualitativa entre as metodologias. Para realmente consolidarmos esta metodologia, há necessidade de implementar o sistema físico para obtermos o resultado de laboratório.

\section{Agradecimentos}

Agradecemos a CAPES pelo apoio financeiro dado a este trabalho. Agradecimento ao Departamento de Engenharia Elétrica da UNESPIlha Solteira, aos orientadores Dr. José Paulo Garcia e Dr. Marcelo Carvalho Minhoto Teixeira.

\section{Referências Bibliográficas}

CRUZ, J. J. da. Controle Robusto Multivariável. EDUSP, 1996.

LEONHARD, W. Control of Electrical Drives. Berlim: Spring Verlag, 1985.

SILVA, G. S. Controle Robusto de Máquinas Assíncronas Usando Estrutura Variável E Modo Deslizantes. 1998. Tese (Mestrado) - UNESP, Ilha Solteira.

RIBEIRO, S. M. J. Controle de Motores de Indução e Cicloconversores Controlados. Ilha Solteira, dez. 1998 Relatório de iniciação científica - UNESP.

TZOU, Ying-Yu. LQG/LTR Control of an AC Induction Servo Drive. IEEE Transactions On Power Electronics, v. 10 , n. 2, mar. 1995. 
